\title{
Bubbling in delay-coupled lasers
}

\author{
V. Flunkert, ${ }^{1}$ O. D’Huys, ${ }^{2}$ J. Danckaert, ${ }^{2,3}$ I. Fischer, ${ }^{4}$ and E. Schöll ${ }^{1}$ \\ ${ }^{1}$ Institut für Theoretische Physik, TU Berlin, Hardenbergstraße 36, 10623 Berlin, Germany \\ ${ }^{2}$ Department of Physics (DNTK), Vrije Universiteit Brussel, Pleinlaan 2, 1050 Brussel, Belgium \\ ${ }^{3}$ Department of Applied Physics and Photon (TONA), Vrije Universiteit Brussel, Pleinlaan 2, 1050 Brussel, Belgium \\ ${ }^{4}$ School of Engineering and Physical Sciences, Heriot-Watt University, Edinburgh, EHI 4AS Scotland, United Kingdom
}

(Received 23 January 2009; published 4 June 2009)

\begin{abstract}
We theoretically study chaos synchronization of two lasers which are delay coupled via an active or a passive relay. While the lasers are synchronized, their dynamics is identical to a single laser with delayed feedback for a passive relay and identical to two delay-coupled lasers for an active relay. Depending on the coupling parameters the system exhibits bubbling, i.e., noise-induced desynchronization, or on-off intermittency. We associate the desynchronization dynamics in the coherence collapse and low-frequency fluctuation regimes with the transverse instability of some of the compound cavity's antimodes. Finally, we demonstrate how, by using an active relay, bubbling can be suppressed.
\end{abstract}

DOI: 10.1103/PhysRevE.79.065201

PACS number(s): 05.45.Xt, 42.55.-f

Synchronization phenomena of coupled nonlinear oscillators are omnipresent and play an important role in physical, chemical, and biological systems [1,2]. Understanding the synchronization mechanisms is crucial for many practical applications. One of the most interesting and challenging phenomena when coupling nonlinear systems is the synchronization of chaotic dynamics [3]. In order to characterize the synchronization effects, stability properties are a key issue. Noise can, for instance, cause intermittent desynchronization. This behavior is called bubbling [4] and has been observed, for example, in optical $[5,6]$ and electrical [7] systems.

Semiconductor lasers are of particular interest in the study of chaos synchronization. The synchronization properties may facilitate new secure communication schemes. However, if two identical semiconductor lasers are optically coupled over a finite distance, it has been observed that the coupling delay leads to spontaneous symmetry breaking, and only generalized synchronization of leader-laggard type occurs [8]. A passive relay in the form of a semitransparent mirror or an active relay in the form of a third laser in between the two lasers has been shown to stabilize the isochronous synchronization solution [9-12], rendering such configurations attractive for chaos-based applications, such as, bidirectional encrypted communication, or chaos-based key exchange, as detailed in Ref. [13].

In this work we show theoretically that bubbling and onoff intermittency occur in both relay setups. In the coherence collapse (CC) and in the low-frequency fluctuation (LFF) regime, we find that bubbling is caused by transversally unstable external cavity modes (ECMs). In the LFF regime the localization of the transversally unstable modes in the synchronization manifold (SM) results in desynchronization during power dropouts, which has also been observed in unidirectionally coupled lasers [14]. For the active relay we find that bubbling can be suppressed by stronger pumping of the relay laser. We consider two identical systems which are delay-coupled via a relay (Fig. 1).

The relay may be an active element or a passive element which merely distributes the arriving signals between the systems. Each system receives a delayed signal from the relay

$$
\dot{\mathbf{X}}_{j}=\mathbf{f}\left(\mathbf{X}_{j}\right)+K \mathbf{Y}(t-\tau / 2) \quad(j=1,2) .
$$

Here $\mathbf{X}_{j}, \mathbf{Y} \in \mathbb{R}^{n}$ are the state vectors of the system $j$ and the relay, respectively, $\mathbf{f}$ is a nonlinear function, $K$ is the relayto-system coupling matrix, and $\tau$ is the propagation delay between system 1 and system 2 . The overdot denotes the derivative with respect to time $t$.

For the active relay we consider the equation

$$
\dot{\mathbf{Y}}=\mathbf{g}(\mathbf{Y})+\frac{1}{2} L \mathbf{X}_{1}(t-\tau / 2)+\frac{1}{2} L \mathbf{X}_{2}(t-\tau / 2),
$$

where $L$ is the system-to-relay coupling matrix and the function $\mathbf{g}$ describes the internal dynamics of the relay. For the passive relay we consider the algebraic equation

$$
\mathbf{Y}(t)=\frac{1}{2}\left[\mathbf{X}_{1}(t-\tau / 2)+\mathbf{X}_{2}(t-\tau / 2)\right] .
$$

Equation (1) together with the relay Eq. (2) or (3) allow for an isochronous (or zero-lag) solution $\mathbf{X}_{1}(t)=\mathbf{X}_{2}(t)$, respectively. The SM is thus invariant. To analyze the stability of this solution we introduce a symmetric variable $\mathbf{S}=\frac{1}{2}\left(\mathbf{X}_{1}+\mathbf{X}_{2}\right)$ and an antisymmetric variable $\mathbf{A}=\frac{1}{2}\left(\mathbf{X}_{1}-\mathbf{X}_{2}\right)$. Equation (1) can then be rewritten in the new variables,

$$
\begin{gathered}
\dot{\mathbf{S}}=\frac{1}{2}[\mathbf{f}(\mathbf{S}+\mathbf{A})+\mathbf{f}(\mathbf{S}-\mathbf{A})]+K \mathbf{Y}(t-\tau / 2), \\
\dot{\mathbf{A}}=\frac{1}{2}[\mathbf{f}(\mathbf{S}+\mathbf{A})-\mathbf{f}(\mathbf{S}-\mathbf{A})] .
\end{gathered}
$$

Note that due to the symmetric coupling the delay terms and all the coupling parameters in Eq. (5) vanish. Equation (5) taken at $\dot{\mathbf{A}}=0$ has a solution $\mathbf{A}=0$ which represents the isochronously synchronized state. Its stability is determined by

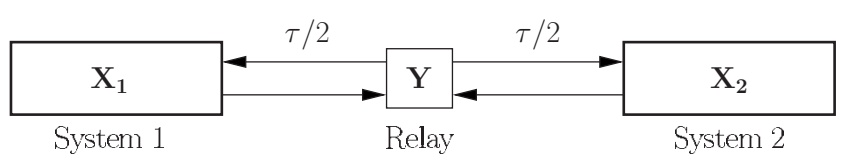

FIG. 1. Schematic setup. 
linearizing Eqs. (4) and (5) in the variable $\mathbf{A}$ around $\mathbf{A}=0$, i.e., we linearize orthogonal to the SM,

$$
\begin{gathered}
\dot{\mathbf{S}}=\mathbf{f}(\mathbf{S})+K \mathbf{Y}(t-\tau / 2), \\
\dot{\mathbf{A}}=D \mathbf{f}(\mathbf{S}) \mathbf{A} .
\end{gathered}
$$

Here, $D \mathbf{f}(\mathbf{S})$ denotes the Jacobian of $\mathbf{f}$ evaluated at position S. Since $\mathbf{S}$ depends on time, Eq. (7) constitutes a timedependent variational equation.

For both relay types the dynamics within the SM resembles the dynamics of a single system with either selffeedback (passive relay)

$$
\dot{\mathbf{S}}=\mathbf{f}(\mathbf{S})+K \mathbf{S}(t-\tau)
$$

or coupling to the active relay

$$
\begin{aligned}
\dot{\mathbf{S}} & =\mathbf{f}(\mathbf{S})+K \mathbf{Y}(t-\tau / 2), \\
\dot{\mathbf{Y}} & =\mathbf{g}(\mathbf{Y})+L \mathbf{S}(t-\tau / 2)
\end{aligned}
$$

In both cases the stability of the synchronized solution is governed by Eq. (7). However, the trajectory $\mathbf{S}(t)$ will be different and the synchronized state may thus have different stability properties.

Bubbling occurs $[4,15]$ when an invariant set $I$, for example, a periodic orbit, in the SM is transversally unstable, while the chaotic attractor in the SM is still transversally stable, i.e., the largest transversal Lyapunov exponent of the attractor is negative, $\lambda_{\perp}<0$. In this situation the trajectory can be pushed toward the unstable set by noise and leave the SM. If there is no other attractor present, the trajectory will eventually come back to the SM and the systems will synchronize again. The point where the invariant set $I$ loses its transverse stability is called bubbling bifurcation, while the point where the attractor itself becomes unstable is called blow-out bifurcation.

For semiconductor lasers the dynamics of each system is governed by the dimensionless Lang-Kobayashi rate equations $[16,17]$

$$
\begin{gathered}
\dot{E}_{j}=\frac{1}{2}(1+i \alpha) n_{j} E_{j}+K e^{i \varphi} E_{\mathbf{Y}}(t-\tau / 2)+F_{j}(t), \\
T \dot{n}_{j}=p-n_{j}-\left(1+n_{j}\right)\left|E_{j}\right|^{2} .
\end{gathered}
$$

Here, $E_{j}$ and $E_{\mathbf{Y}}$ are the complex electric field amplitudes of the $j$ th system and the relay, respectively, $n_{j}$ is the excess carrier density, $\alpha$ is the linewidth enhancement factor, $p$ is the pump current, and the time scale parameter $T=\tau_{c} / \tau_{p}$ is the ratio of the carrier $\left(\tau_{c}\right)$ and the photon $\left(\tau_{p}\right)$ lifetime. For simplicity we choose the feedback phase $\varphi=0$. Note that in general one could also include coupling phases in Eq. (3). This leads to interference conditions of all phases which have to be satisfied for isochronous synchronization. In our simulations we consider the spontaneous emission noise via a complex Gaussian white random variable $F_{j}(t)$ with the covariance $\left\langle F_{j}(t) F_{i}\left(t^{\prime}\right)^{*}\right\rangle=\beta\left(n+n_{0}\right) \delta_{i j} \delta\left(t-t^{\prime}\right)$, where $n_{0}=10$ is the carrier density at threshold and $\beta=10^{-5}$ is the spontaneous emission factor. Carrier noise has not been taken into account at this level.

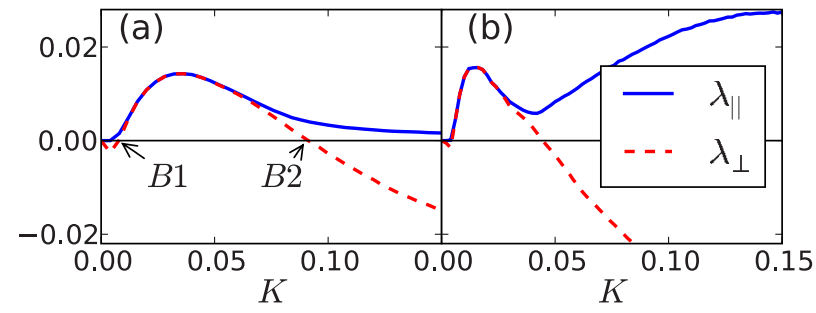

FIG. 2. (Color online) Maximum transversal Lyapunov exponent $\lambda_{\perp}$ (red dashed) and maximum parallel Lyapunov exponent $\lambda_{\|}$ (blue solid) as a function of the feedback strength $K$ for (a) passive relay and (b) active relay $\left(p_{\text {relay }}=4.0\right)$. At the two blow-out bifurcations $B 1$ and $B 2$ the maximum transversal Lyapunov exponent of the chaotic attractor changes sign. Other parameters: $T=200$, $p=1.0, \tau=1000, \alpha=4, \varphi=0$

If the relay is realized through a semitransparent mirror (passive relay), the dynamics within the SM is given by Eq. (11) with $E_{\mathbf{Y}}(t-\tau / 2)=E_{j}(t-\tau)$, i.e., an effectively decoupled laser. For this configuration we calculate the maximum parallel Lyapunov exponents $\lambda_{\|}$(within the SM) as well as the maximum transversal Lyapunov exponents $\lambda_{\perp}$ by simulating the dynamics in the SM without noise and applying the method developed in [18]. Figure 2(a) displays the Lyapunov exponents as a function of the feedback strength $K$. There are two blow-out bifurcations [19] at $K \approx 0.008(B 1)$ and at $K \approx 0.09(B 2)$, where $\lambda_{\perp}$ changes sign and the chaotic attractor loses its transversal stability. Similar behavior is found for an active relay [Fig. 2(b)].

Over a wide range of $K$ [Fig. 2(a)], in which the attractor is stable and the dynamics is chaotic, we observe bubbling induced by spontaneous emission noise. In these regimes, when the noise is switched off in the simulations, the two lasers stay perfectly synchronized. In the regime with $\lambda_{\perp}>0$ we observe desynchronization bursts even without noise, i.e., the system exhibits on-off intermittency. Figure 3(a) depicts the bubbling behavior for values of $K$ above $B 2$ where the laser operates in the $\mathrm{CC}$ regime. Figure $3(\mathrm{~b})$ corresponds to a lower pump current, where the synchronized lasers operate in the LFF regime. In this regime bubbling only takes place during the power dropouts. In both cases, when the noise amplitude is decreased, the desynchronization peaks occur less frequently; the maximum height, however, does not decrease.

We now relate the desynchronization dynamics to the transverse stability of the ECMs in the SM. These modes organize the dynamics in the SM in the CC and the LFF regime. The ECMs are rotating wave solutions of the form $E(t)=A \exp (i \omega t)$ and $n(t)=n$ with constant values $A, \omega$, and $n$. They are well-studied [20] solutions of the LangKobayashi equations and are located on an ellipse in the $(\omega, n)$ plane [see inset of Fig. 4(a)]. The modes on the top and bottom half of the ellipse are called modes and antimodes, respectively.

The transverse stability of an ECM is governed by the variational Eq. (7) where $\mathbf{S}(t)$ is the ECM solution. To determine the stability, we transform the laser equations into a rotating frame $[21] E_{0}=E \exp (-i \omega t)$. In these coordinates, an $\mathrm{ECM} E=A \exp (i \omega t+i \psi)$ is transformed into a family of fixed 

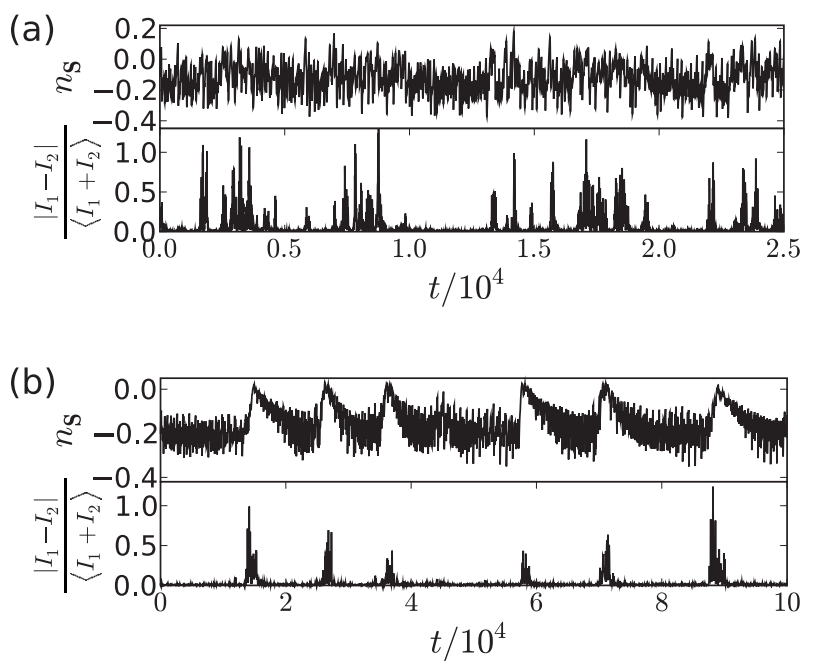

FIG. 3. Carrier density of the symmetric variable $n_{\mathbf{S}}=\frac{1}{2}\left(n_{1}+n_{2}\right)$ and intensity difference $\left|I_{1}-I_{2}\right| /\left\langle I_{1}+I_{2}\right\rangle$ (normalized by the mean intensity) representing the deviation from the synchronized state vs time. (a) Bubbling in the coherence collapse regime $(p=1.0)$. (b) Bubbling in the low-frequency fluctuation regime during power dropouts $(p=0.1)$. Other parameters: $T=200, K=0.12$, $\tau=1000, \alpha=4$

points $E_{0}=A \exp (i \psi)$. Splitting the complex electric field $E_{0 j}=x_{j}+i y_{j}$ and using the vector $\mathbf{X}_{j}=\left(x_{j}, y_{j}, n_{j}\right)$, Eq. (11) can be written in the form of Eq. (1) and the above analysis applies. The eigenvalues of the Jacobian in the rotating frame then determine the ECM transverse stability. Figure 4(a) displays the position of the ECMs in the $(\omega, n)$ plane and their stability for a choice of parameters. The black trajectory displays the projection of the symmetric variable $n_{\mathbf{S}}$.

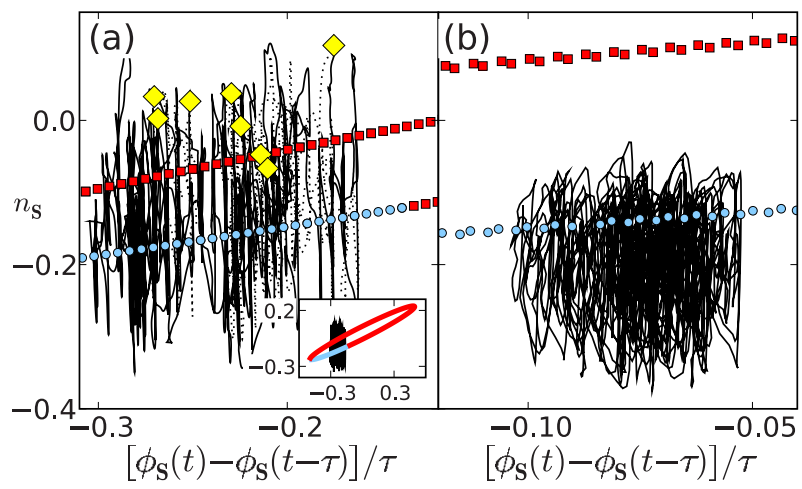

FIG. 4. (Color online) Projection of the dynamics of the symmetrized solution $n_{\mathbf{S}}, E_{\mathbf{S}}=A_{\mathbf{S}} \exp \left(i \phi_{\mathbf{S}}\right)$ (black trajectory) onto the $(\omega, n)$ plane for (a) passive relay $(p=1.0)$ and (b) active relay $\left(p=1.0, p_{\text {relay }}=4.0\right)$. Transversally stable (blue circles) and transversally unstable (red squares) ECMs are also shown. (a) The competition between chaotic itinerancy and antimodes leads to bubbling during global antimode dynamics. Yellow diamonds mark the onset of desynchronization. Solid and dashed parts of the trajectory correspond to synchronized and desynchronized periods, respectively. The inset in (a) shows the ECM ellipse and bubbling dynamics in a larger range. (b) The system evolves around the transversally stable compound laser modes and bubbling is suppressed. Parameters are as in Fig. 3.
The bubbling behavior in the $\mathrm{CC}$ regime and the correlation of the desynchronization with the power dropouts in the LFF regime can be understood as follows. In the CC regime the dynamics comprises chaotic itinerancy among the modes and global antimode dynamics [22] [see Fig. 4(a)]. The modes involved in the chaotic itinerancy are transversally stable (blue circles). The antimodes on the other hand are transversally unstable (red squares). Thus, when the trajectory approaches the antimode, noise can lead to desynchronization and bubbling occurs. The yellow diamonds in Fig. 4(a) mark the onset of desynchronization, showing that bubbling always occurs in the vicinity of the antimodes (independent of the power). Please note that due to the role of noise not every approach to an antimode results in a bubbling excursion.

In the LFF regime [23] the dynamics is similar. The intensity buildup process in between power dropouts is characterized by chaotic switching between different attractor ruins (ghosts) of unstable ECMs with a drift toward the ECM with minimal $n$. All ECMs involved in the buildup process are transversally stable and we observe no desynchronization. After a transient time, a power dropout takes place. During the dropout the trajectory collides with an antimode in a crisis. Again, the vicinity to transversally unstable antimodes-rather than the drop in power-leads to bubbling behavior.

The transverse stability of the ECMs depends on the laser and coupling parameters as well as on the parameters of the particular ECM. Note that modes and antimodes are not necessarily transversally stable or unstable, respectively. The modes on the lower right-hand side of Fig. 4(a), for instance, are transversally unstable. With decreasing coupling strength $K$, more modes become transversally unstable until the whole chaotic attractor loses its transversal stability. This leads to the blowout bifurcation $B 2$ in Fig. 2.

With increasing feedback strength the bubbling occurs less frequently and the average synchronization interval $\Delta$ increases; however, we did not find a transition to a bubbling-free state in a physically reasonable range of $K$. Note that neither $K$ nor the other parameters of our model are normal parameters in the sense of Ref. [5]. Thus we do not observe power-law scaling of $\Delta$ as in $[6,15]$. The parallel Lyapunov exponent $\lambda_{\|}$approaches zero with increasing $K$ and the chaoticity decreases, making this situation less interesting for chaos-based applications.

If the elements are coupled via an active relay, the synchronized lasers behave similar to two delay-coupled lasers [see Eq. (9)]. If we choose $\mathbf{f}=\mathbf{g}$ and $K=L$, we obtain a system of two identical mutually coupled semiconductor lasers, which has been studied before $[8,24,25]$. Such a system has rotating wave solutions of the form $E_{\mathbf{S}}(t)=A_{\mathbf{S}} \exp (i \omega t)$, $E_{\mathbf{Y}}(t)=A_{\mathbf{Y}} \exp (i \omega t+i \psi), \quad n_{\mathbf{S}}(t)=n_{\mathbf{S}}$, and $n_{\mathbf{Y}}(t)=n_{\mathbf{Y}}$, called compound laser modes (CLMs). Their spectrum is more complex than for the ECMs; besides the synchronized solutions (which correspond to ECMs), there exist antisymmetric modes, for which the relay and the synchronized solution are in antiphase $(\psi=\pi)$, as well as asymmetric modes where the relay has a different intensity than the outer lasers.

The positions of the transversally unstable modes are close to those of the ECMs of a single laser in the $(\omega, n)$ 
parameter space. Also the dynamics of three identical coupled lasers is similar to the behavior in the presence of a passive relay. Indeed, we find bubbling in both the LFF and $\mathrm{CC}$ regimes.

In the experiments reported in [12] all the coupling parameters in the setup are chosen identical, i.e., $L=2 K$ in Eqs. (1) and (2). But also in this case we observe qualitatively similar laser dynamics, with a trajectory in parameter space coming close to the transversally unstable CLMs.

To suppress the bubbling while maintaining strong chaos, we apply a sufficiently higher pump current to the relay laser $\left(p_{\text {relay }}=4.0\right)$ than to the outer lasers $(p=1.0)$. For this configuration we have calculated $\lambda_{\|} \approx 0.026$ and $\lambda_{\perp} \approx-0.032$, confirming that the system is in the chaotic regime [cf. Fig. 2(b)]. The system still itinerates among the compound laser modes, but there is no global antimode dynamics. Moreover, in contrast to the behavior for the symmetric case $p_{\text {relay }}=1.0$, the active relay now suppresses the bubbling and there is no desynchronization [see Fig. 4(b)]. Inspecting Fig. 4(b), we can conclude that the CLMs involved in the dynamics are indeed transversally stable. If the middle laser is pumped less strongly than the outer ones, the opposite effect is observed.

In conclusion, we have demonstrated a mechanism for desynchronization by bubbling in a very general setting of two delay-coupled lasers with either passive or active relay. We have shown that in the CC and LFF regimes the occurrence of bubbling is related to the transverse instability of some of the compound cavity's antimodes and that, by tuning of the active relay, it is possible to suppress the bubbling. These synchronization properties are decisive for the setup of chaos-synchronization-based applications and provide a strategy on how to achieve stable synchronization.

We thank P. Ashwin, T. Gavrielides, and C. Mirasso for fruitful discussions. O.D. acknowledges the Research Foundation Flanders (FWO-Vlaanderen) for financial and project support. This work was partially supported by the Belgian Science Policy Office under Grant No. IAP-VI10 "photonics@be," by the EC Project GABA FP6-NEST under Contract No. 043309, and by DFG in the framework of Sfb 555 .
[1] S. Boccaletti, J. Kurths, G. Osipov, D. L. Valladares, and C. S. Zhou, Phys. Rep. 366, 1 (2002).

[2] A. Pikovsky, M. G. Rosenblum, and J. Kurths, Synchronization: A Universal Concept in Nonlinear Sciences (Cambridge University Press, Cambridge, England, 2001).

[3] L. M. Pecora and T. L. Carroll, Phys. Rev. Lett. 64, 821 (1990).

[4] P. Ashwin, J. Buescu, and I. Stewart, Phys. Lett. A 193, 126 (1994).

[5] J. R. Terry, K. S. Thornburg, D. J. DeShazer, G. D. VanWiggeren, S. Zhu, P. Ashwin, and R. Roy, Phys. Rev. E 59, 4036 (1999).

[6] M. Sauer and F. Kaiser, Phys. Lett. A 243, 38 (1998).

[7] D. J. Gauthier and J. C. Bienfang, Phys. Rev. Lett. 77, 1751 (1996).

[8] J. Mulet, C. R. Mirasso, T. Heil, and I. Fischer, J. Opt. B: Quantum Semiclassical Opt. 6, 97 (2004).

[9] L. B. Shaw, I. B. Schwartz, E. A. Rogers, and R. Roy, Chaos 16, 015111 (2006).

[10] E. Klein, N. Gross, M. Rosenbluh, W. Kinzel, L. Khaykovich, and I. Kanter, Phys. Rev. E 73, 066214 (2006).

[11] A. S. Landsman and I. B. Schwartz, Phys. Rev. E 75, 026201 (2007).

[12] I. Fischer, R. Vicente, J. M. Buldú, M. Peil, C. R. Mirasso, M.
C. Torrent, and J. García-Ojalvo, Phys. Rev. Lett. 97, 123902 (2006).

[13] R. Vicente, C. R. Mirasso, and I. Fischer, Opt. Lett. 32, 403 (2007).

[14] V. Ahlers, U. Parlitz, and W. Lauterborn, Phys. Rev. E 58, 7208 (1998).

[15] S. C. Venkataramani, B. R. Hunt, E. Ott, D. J. Gauthier, and J. C. Bienfang, Phys. Rev. Lett. 77, 5361 (1996).

[16] R. Lang and K. Kobayashi, IEEE J. Quantum Electron. 16, 347 (1980).

[17] P. M. Alsing, V. Kovanis, A. Gavrielides, and T. Erneux, Phys. Rev. A 53, 4429 (1996).

[18] J. Doyne Farmer, Physica D 4, 366 (1982).

[19] E. Ott and J. C. Sommerer, Phys. Lett. A 188, 39 (1994).

[20] J. Mørk, B. Tromborg, and J. Mark, IEEE J. Quantum Electron. 28, 93 (1992).

[21] S. Yanchuk, Math. Methods Appl. Sci. 28, 363 (2005).

[22] J. Mulet and C. R. Mirasso, Phys. Rev. E 59, 5400 (1999).

[23] T. Sano, Phys. Rev. A 50, 2719 (1994).

[24] H. Erzgräber, D. Lenstra, B. Krauskopf, E. Wille, M. Peil, I. Fischer, and W. Elsäßer, Opt. Commun. 255, 286 (2005).

[25] H. Erzgräber, B. Krauskopf, and D. Lenstra, SIAM J. Appl. Dyn. Syst. 5, 30 (2006). 